\title{
Cure depths with LED light curing units
}

\author{
Optical power outputs, spectra and dental composite depths of cure, obtained with blue light emitting diode (LED) \\ and halogen light curing units (LCUs) R. W. Mills, A. Uhl and K. D. Jandt Br Dent J 2002; 193: 459-463
}

\section{Objective}

To test the hypothesis that a prototype LED light curing unit, (LCU), a commercial LED LCU and a halogen LCU achieve similar cure depths, using two shades of a camphorquinone photoinitiated dental composite. To measure the LCUs' outputs and the frequency of the LED LCU's pulsed light, using a blue LED array as a photodetector.

Design

Cure depth and light output characterisation to compare the LCUs.

Setting

An in vitro laboratory study conducted in the UK.

Materials and Methods

The LCUs cured A2 and A4 composite shades. A penetrometer measured the depth of cure. Analysis was by one-way ANOVA, two-way univariate ANOVA and Fisher's LSD test with a 95\% confidence interval. A power meter and spectrograph characterised the LCUs' emissions. A blue LED array measured the pulsed light frequency from an LED LCU.

Results

Statistically significant different LCU irradiances $\left(119 \mathrm{~mW} / \mathrm{cm}^{2}\right.$ to $851 \mathrm{~mW} / \mathrm{cm}^{2}$ ) and cure depths ( $3.90 \mathrm{~mm} \mathrm{SD} \pm 0.08$ to $6.68 \mathrm{~mm} \mathrm{SD}$ $\pm 0.07)$ were achieved. Composite shade affected cure depth. A blue LED array detected pulsed light at $12 \mathrm{~Hz}$ from the commercial LED LCU.

Conclusions

The prototype LED LCU achieved a greater or equal depth of cure when compared with the commercial LCUs. LEDs may have a potential in dentistry for light detection as well as emission.

\section{IN BRIEF}

- Advances in the power output of light emitting diodes (LEDS) have allowed a LED light curing unit (LCU) with an $8 \mathrm{~mm}$ diameter light guide tip to achieve a similar depth of cure in a camphorquinone photoinitiated composite, as a halogen LCU.

- LEDs have long lifetimes and a more consistent output than halogen bulbs. The power density (irradiance) of an LED LCU does not have to be as high as a conventional halogen LCU to achieve the same depth of cure.

- Blue LEDs can be used as photodetectors and may be of future use in dental radiometers.

- When assessing the output from an LCU it is not only important to know the spectrum and irradiance, but also whether the emission is pulsed or continuous.

\section{COMMENT}

The properties of light activated resin-composites are significantly affected by the light intensity of curing units. New types of curing units have been introduced into the market. ${ }^{1}$ This study focuses on blue light emitting diodes as a viable alternative curing unit. The authors designed and synthesized a prototype LED composed of 63 LEDs. This was compared with a commercial LED composed of 7 LEDs, and a conventional halogen-curing unit. The output of all units was measured with a power meter. Their effect on depth of cure was evaluated with a penetrometer on two shades (A2 and A4) of a commercially available hybrid composite.

The results showed that the prototype LED had a lower power output compared with the halogen unit but higher than the commercial LED. However, the prototype LED produced slightly higher values for depth of cure compared with the halogen unit, whilst the commercial LED had the lowest values and was the only one that did not reach an adequate level of polymerisation for the darker shade composite. As the authors point out this is in agreement with previous studies and can be explained by the effectiveness of different spectral outputs. ${ }^{2}$

As it is indicated by the results the intensity of an LED can vary and significantly affect the extent of polymerisation of the material. Also the chemistry of the materials is critical and more specifically that of their initiators. If the initiator absorbs outside the narrow emission of the LED the restoration might be compromised. Other properties of the materials should be studied in the future like mechanical properties and the extent of cure. However, it is evident that blue LED technology continues to improve and can be very useful in the field of dentistry. Its advantages of reliability, handling and hygiene justify the need for further research on the subject. ${ }^{3}$

Nick Silikas, Research Associate, Biomaterials Unit, Manchester Dental School

1. Davidson C L, de Gee A J. Light-curing units, polymerization, and clinical implications. JAdhes Dent 2000; 2:167-173.

2. Fan P L, Schumacher R M, Azzolin K, Geary R, Eichmiller F C. Curing-light intensity and depth of cure of resin-based composites tested according to international standards. J Am Dent Assoc 2002; 186: 568-572.

3. Althoff 0, Hartung M. Advances in light curing. Am J Dent, 2000; 13: 77D-81D. 